\title{
Criminologie
}

\section{Sang de belette et cervelle de corbeau : la médicalisation de la folie au Québec, 1600-1850}

\section{André Cellard}

Volume 26, numéro 1, 1993

Michel Foucault et la (post) modernité

URI : https://id.erudit.org/iderudit/017334ar

DOI : https://doi.org/10.7202/017334ar

Aller au sommaire du numéro

Éditeur(s)

Les Presses de l'Université de Montréal

ISSN

0316-0041 (imprimé)

1492-1367 (numérique)

Découvrir la revue

Citer cet article

Cellard, A. (1993). Sang de belette et cervelle de corbeau : la médicalisation de la folie au Québec, 1600-1850. Criminologie, 26(1), 165-175.

https://doi.org/10.7202/017334ar
Résumé de l'article

Like most areas of health that interested medicine in the 19th century, it was almost without opposition that insanity was to become a new medical specialty during the past century. The aim of this article is to shed some light on the dynamics that have allowed doctors since the I7tl\% and 18th century to share their point of view with the general public for whom the existential causes of madness seem to have been taken for granted. 


\section{SANG DE BELETTE ET CERVELLE DE CORBEAU : LA MÉDICALISATION DE LA FOLIE AU QUÉBEC, 1600-1850 ${ }^{1}$ André Cellard ${ }^{2}$}

Like most areas of health that interested medicine in the $19^{\text {th }}$ century, it was almost without opposition that insanity was to become a new medical specialty during the past century. The aim of this article is to shed some light on the dynamics that have allowed doctors since the $17^{\text {th }}$ and $18^{\text {th }}$ century to share their point of view with the general public for whom the existential causes of madness seem to have been taken for granted.

Du guérisseur tribal faisant appel aux forces de l'au-delà jusqu'au spécialiste moderne qui, lui, tire son pouvoir d'une technologie inaccessible pour le commun des mortels, peu de professions ont, dans l'histoire, joui d'un ascendant et d'un prestige social aussi tangible que celle de médecin. À cet égard, le Québec ne fait pas exception, bien au contraire, puisque la profession médicale fut longtemps, avec celle d'avocat, le débouché par excellence de l'élite canadienne-française instruite.

Si la médecine officielle exerce désormais un monopole presque inébranlable sur le marché de la santé, c'est qu'elle sut s'imposer définitivement aux «usurpateurs» et aux «charlatans» de tout acabit au siècle dernier. La légitimité de cette lutte et de cette victoire a toujours reposé sur la scientificité dont se réclamait le corps médical. Depuis Hippocrate, en effet, des générations de médecins n'avaient-elles pas cherché à percer les secrets de la nature et, bâtissant toujours plus haut sur les bases solides de leurs devanciers, de progrès en progrès, soulagé toujours plus efficacement l'humanité souffrante?

Évidemment, il n'a jamais fait aucun doute que la science ne pouvait que relever de la culture savante, celle de l'élite, et qu'il n'y avait qu'elle de valable. C'est pourquoi l'on a conservé si peu de la culture médicale populaire, par exemple, et on ne peut que se réjouir de voir, depuis quelques années, les historien(ne)s se pencher sur l'étude de la médecine dite populaire.

1. L'auteur tient à remercier M. Jacques Laplante du département de Criminologie de l'Université d'Ottawa pour sa lecture attentive de ce texte.

2. L'auteur est professeur au département de Criminologie de l'Université d'Ottawa, 1, Stewart St., Ottawa, Ontario, Canada, K1N 6N5. 
En effet, la population en général n'était pas nécessairement cette masse ignorante et aveugle qui devait se laisser guider par les lumières de la science et du progrès. Elle pouvait, elle aussi, relier les effets aux causes et il y a peut-être autant à apprendre de ceux qui fondaient leurs connaissances sur des siècles d'observation et de pratique quotidienne que sur les élucubrations souvent prétentieuses de ceux qui croyaient détenir le monopole de la vérité. Dernièrement, l'histoire nous a ainsi permis de redécouvrir certaines dimensions du savoir populaire entre autres au niveau des accouchements, de la régulation et du contrôle des naissances et des médecines douces. À cet égard, l'histoire de la folie au Québec vient elle aussi jeter un peu de lumière sur la question de l'opposition entre le savoir populaire et celui de l'élite du XVII ${ }^{\mathrm{e}}$ au $\mathrm{XIX}^{\mathrm{e}}$ siècles.

\section{LES CAUSES DE LA FOLIE AU XVII ${ }^{\mathrm{e}}$ SIÈCLE}

Les causes surnaturelles: la compréhension d'une intervention donnée à l'égard d'un fou passe nécessairement par celle des causes présumées du mal dont il souffre. C'est ainsi que dans l'historiographie occidentale on a souvent prétendu qu'à la Renaissance, bon nombre de fous et de folles surtout étaient montés sur le bûcher ou avaient subi l'exorcisme, laissant ainsi entendre que la possession démoniaque était généralement perçue comme cause principale de la folie. Les choses ainsi présentées, les premiers tâtonnements des médecins en matière de santé mentale paraissent héroïques et salvateurs face aux conséquences affreuses de l'obscurantisme généralisé.

Au Québec, pour ne pas être en reste, on a raconté que l'exorcisme avait aussi été le lot des malheureux fous et folles de la Nouvelle-France au $\mathrm{XVII}^{\mathrm{e}}$ siecle $^{3}$. Un cas unique vient appuyer une telle thèse, celui de Barbe Hallé, une jeune fille de Beauport que les Jésuites croyaient, en 1660 . possédée d'un sorcier. On voyait voler des pierres, jouer de la flûte et du tambour dans sa maison et c'est pourquoi il fut décidé qu'elle serait exorcisée ${ }^{4}$.

3. H. Wallot, «Perspectives sur l'histoire québécoise de la psychiatrie: le cas de l'asile de Québec», Santé mentale au Québec, vol. IV, nº 1 (juin 1979), p. 103; M. Meloche, «Enfermer la folie», Santé mentale au Québec, vol. VI, n² 2 (nov. 1981), p. 17; R. Viau, L'image de la folie dans le roman québécois, thèse de doctorat, Lettres françaises, Université d'Ottawa, 1984, p.74-75; et F. Boudreau, qui titre un chapitre: «De l'exorcisme aux loges» dans De l'asile d̀ la santé mentale, Montréal, St-Martin, 1984, p. 21.

4. Marie de L'Incarnation, Lettres de la Révérende Mère Marie de l'Incarnation, Tournai, Casterman, 1876, vol. 2, p. 208 et 377. 
Nous nous expliquons assez difficilement le choix unanime de cette anecdote afin d'illustrer l'attitude de la population face à la folie puisque jamais, dans toute cette histoire, en fut-il question ou même fait allusion. En effet, il ne s'agit ici que de quelques membres du clergé qui ont conclu à un cas de possession parce qu'ils étaient supposément témoins de phénomenes surnaturels dans sa demeure. C'est pour cette seule raison que la jeune fille fut exorcisée. On ne l'a jamais cru folle, sa «folie» lui étant plutôt attribuée par nos contemporains ${ }^{5}$.

Fort heureusement, l'histoire de la déviance mentale au XVII ${ }^{\mathrm{e}}$ siècle ne se limite pas ici à ce cas pour le moins douteux, et des recherches plus approfondies permettent d'esquisser, pour cette période, une histoire de la folie plus complexe et étoffée ${ }^{6}$. En effet, les fous étaient bel et bien présents dans le tissu quotidien en Nouvelle-France et c'est dans les sources du quotidien: annales, récits, lettres. relations, documents notariaux, entre autres, qu'on les retrouve au fil du hasard.

Pour les habitants de la colonie française, la folie, pas plus que toute autre maladie, n'était causée par l'action de Satan. Elle résultait plutôt d'un malfonctionnement du cerveau, "siège de l'esprit ${ }^{7}$ ", mauvais fonctionnement attribuable à deux types de causes de nature différente selon le niveau de culture de celui ou celle qui s'exprimait ainsi sur l'origine du mal.

Les causes de nature physiologique: pour les individus possédant quelque culture savante, la folie, comme d'ailleurs toute autre maladie, était expliquée par la théorie des humeurs. Remontant à Hippocrate et très en vogue parmi le corps médical depuis la Renaissance, la théorie des humeurs postulait que la masse liquide du corps humain était composée de quatre éléments ou humeurs soit: le sang, la bile jaune, la bile noire et le phlegme (pituite). C'est le déséquilibre, le bouleversement des humeurs dans l'organisme qui provoquait, au dire des «savants», les différentes maladies. Mises en effervescence par le processus de digestion, par exemple, les humeurs pouvaient ainsi se refroidir ou au contraire surchauffer, permettant à des substances ou à des vapeurs nocives de se concentrer au cerveau, provoquant des troubles tels la mélancolie (dépression), la manie, la frénésie, l'épilepsie, ou tout autre trouble de l'organe de la pensée. La mélancolie, à

5. Il s'agirait même đ'une "psychopathe" selon le diagnostic de M. Meloche, 1981, p. 103.

6. A. Cellard, Histoire de la folie au Québec. 1600-1850, Montréal, Boréal, 1991. Voir aussi: A. Paradis et al, Essais pour une préhistoire de la psychiatrie au Canada, 1800-1880, Université du Québec à Trois-Rivières, 1977, coll. «Recherches et Théories ", $n^{\circ} 15$.

7. Les Relations des Jésuites, Québec, A. Côté éd., 1858, vol. 3, (1656), p. 15. 
titre d'exemple, était provoquée par une accumulation de bile noire au cerveau («être d'une humeur noire») alors que la frénésie, elle, pouvait résulter de vapeurs chaudes issues de fièvres ${ }^{8}$.

C'est par cette thérie «savante » que l'élite instruite expliquait les cas de folie dont elle était témoin. C'est ainsi que la Supérieure de la communauté de l'Hôtel-Dieu de Montréal en arrive à la conclusion qu'il y a «des vapeurs mêlées dans (la) folie» d'une consœur ${ }^{9}$. Le jésuite Sagard dit de vieillards que leur cerveau est parfois «trop chargé d'humeur et de phlegme ${ }^{10} »$. D'autres membres de la Compagnie de Jésus font aussi mention d'individus devenus fous parce que «travaillé(s) par quelque fièvre chaude» ou «tombés en frénésie par la violence de la fièvre ${ }^{11}$.

La cause de nature «existentielle»: selon Michael McDonald, qui a fait usage d'une documentation exceptionnelle dans son étude de la folie en Angleterre au $\mathrm{XVII}^{\mathrm{e}}$ siècle, la population était à cette époque aussi convaincue que nous que le stress psychologique et social pouvait perturber l'esprit et la santé de l'individu ${ }^{12}$. Plus convaincue que nous le sommes nousmêmes, aurait pu ajouter l'auteur anglais. En effet, cette croyance était très répandue en Nouvelle-France chez les gens ordinaires et se trouve à la base de l'explication donnée à la majorité des troubles mentaux que nous avons retracés pour cette période. L'on considérait que les passions, les émotions fortes, les souffrances morales étaient susceptibles d'altérer le cerveau et de déclencher le désordre mental.

C'est le cas par exemple de $\mathbf{S}^{r}$ Daveluy, qui «a perdu l'esprit tout d'un coup par une imagination des plus fortes qu'elle se fit qu'on voulait la faire sœur domestique ce qui lui faisait une peine extraordinaire ${ }^{13}$ » ou de $S^{r}$ Chevalier qui «devint si excessivement dévote que l'esprit lui tourna ${ }^{14}$ ». Une autre personne «avait de grandes faiblesses d'esprit que l'on attribuait à sa peur qu'elle avait eue en venant de France ${ }^{15}$ ». On blâmait ainsi, afin

8. J. Céard, «Entre le naturel et le démoniaque», Nouvelle Histoire de la Psychiatrie, Toulouse, Privat, 1984, p. 81.

9. M. Morin, Les annales de l'Hôtel-Dieu de Montréal, 1659-1725, Montréal, Presses de l'Université de Montréal, 1979, p. 284.

10. F.G. Sagard, Histoire du Canada et Voyages, Paris, C. Sorniers, 1686, p. 603.

11. Relations des Jésuites, vol. 1 (1634), p. 69, et p. 21.

12. M. McDonald, Mystical Bedlam, Cambridge, Cambridge University Press, 1981, p. 72 .

13. M. Morin, op. cit. p. 72 .

14. Juchereau de Saint Ignace et Duplessis de Sainte Hêlène, Annales de l'HôtelDieu de Québec, 1656-1717, Montréal, Presses de Garden city, 1959, p.45-46.

15. Ibid, p. 75 . 
d'expliquer le désordre mental, d'autres problèmes de nature existentielle telles les peines sentimentales, la perte d'êtres chers et ainsi de suite ${ }^{16}$.

Il est vraiment primordial pour le chercheur de tenter de saisir ainsi la nature des causes présumées de la folie puisqu'elles servaient, et d'ailleurs servent encore, à la détermination du type d'intervenant - prêtres, médecins ou toute autre personne - qui aurait autorité sur elle de même que la forme de traitement que l'on ferait subir à l'individu qui en était atteint.

Ainsi, si l'on était tenant de la théorie des humeurs, c'est à la médecine officielle que l'on faisait appel afin qu'elle puisse rétablir l'équilibre perturbé. Le médecin, puisqu'il croyait le mal d'origine physiologique, appliquait une thérapeutique de nature biologique. Il pouvait alors appliquer au mal quelques techniques de base: saignée, purgation, administration de toniques fortifiants, calmants, humectants et autres. Il lui arrivait aussi d'utiliser des remèdes plus spécifiques à la folie, tels la ciguë ou l'hellébore, utilisés à cette fin depuis l'Antiquité classique et cultivés en Nouvelle-France ${ }^{17}$.

Cependant, les médecins cherchaient aussi le remède miracle, la potion magique qui viendrait à coup sûr à bout de la folie. Ils prescrivaient des vers de terre, des cerveaux de corbeaux ou encore la bile fraîche d'un chien noir. Dans les traités de médecine que possédait l'Hôtel-Dieu de Québec au $\mathrm{XVII}^{\mathrm{e}}$ et début du XVIII ${ }^{\mathrm{e}}$ siècle, on recommande par exemple de traiter le mal caduc (l'épilepsie) au moyen du «foie ou gros boyau d'un loup tué à la chasse», du sang d'une belette, du fiel de la bourse d'un chat noir, de la fiente de pourceau et autres décoctions aussi imaginatives que peu ragoûtantes ${ }^{18}$.

La découverte de nouveaux continents n'avait fait qu'ajouter au nombre de ces thérapeutiques fantaisistes ou pour le moins improvisées et on continua dans l'exotisme la quête $d u$ remède miracle. Au Québec, les ressources nouvelles du milieu et l'apport de la médecine amérindienne allaient fournir l'occasion d'une certaine spécificité à la thérapeutique néofrançaise. C'est ainsi que les rognons de castor - auxquels, d'ailleurs, s'intéressa particulièrement Sarrazin qui produisit sur cet animal un

16. Ibid. p. 83-84; p. 213; M. Morin, Les annales de l'Hôtel-Dieu de Montréal, op.cit, p. 143; Jugements et Délibérations du Conseil Souverain de la Nouvelle-France, Québec, A. Côté, 1885-1891, avril, p. 304-305.

17. P. Boucher, Histoire véritable et Naturelle... (1664), Société Historique de Boucherville, 1964, p. 86.

18. Sur toute cette question voir: A. Cellard, Folie et société au Québec, de la Nouvelle-France au milieu du $19^{e}$ siècle, thèse de Ph.D., Université d'Ottawa, 1988, p. 100-114. 
mémoire à l'académie des Sciences de Paris ${ }^{19}$ - et le sabot d'élan devinrent fort recherchés par les apothicaires d'Europe et firent en NouvelleFrance l'objet d'un commerce fort compétitif ${ }^{20}$.

En ce qui a trait à la population en général, pour qui le désordre mental était plutôt d'origine existentielle, l'approche thérapeutique était plus simple. On semblait considérer que le problème avait pu couver durant longtemps et qu'on ne pouvait le régler d'un coup de baguette ou à l'aide de quelque potion magique. Les gens avaient ainsi tendance à intervenir au niveau du mal de vivre l'origine du désordre. On parle donc ici d'attitudes, de comportements à l'égard des fous.

Dans certains cas, on intervient en éloignant la personne affectée des passions qui l'ont perturbée, on rassure, on réconforte ${ }^{21}$. Un cas intéressant est celui de $S^{r}$ Chevalier, dont «l'esprit avait tourné». Les religieuses avaient décidé à cette occasion de l'envoyer «à Beauport chez $\mathbf{M}^{\mathrm{r}}$ Giffard, notre bon ami, croyant que l'air de la campagne lui serait bon». Là, on «tâcha de la divertir» et «elle se remit si parfaitement que jamais elle ne s'en est sentie, et son esprit a toujours été depuis dans une très bonne assiette $^{22}$ ". Cette thérapeutique n'est pas en effet sans rappeler certains aspects de l'approche «psychologique» que mit sur pied le Français Philippe Pinel plus d'un siècle et demi plus tard. Nous y reviendrons.

Au XVII ${ }^{e}$ siècle, en somme, la médicalisation de la folie relève plutôt de la culture savante et reste marginale par rapport à l'explication d'ordre existentiel qui semble aller de soi pour la plus grande partie de la population.

\section{LES CAUSES DE LA FOLIE, DU XVIII ${ }^{\mathrm{e}}$ SIÈCLE AU MILIEU DU XIX ${ }^{\mathrm{e}}$ SIÈCLE}

Au rythme de la diffusion de la culture de l'élite et à mesure que la médecine officielle gagnait en crédibilité, on constate, cependant, au siècle suivant un recours de plus en plus fréquent au médecin. Mise en confiance par les progrès tangibles réalisés par le corps médical au XVIII ${ }^{\mathbf{e}}$ siècle, la population emboîte le pas à l'explication dite scientifique au détriment, si l'on peut dire, de l'explication «psychologique» de la folie.

19. Ibid., p. 112.

20. Voir la correspondance de Mère de Sainte-Hélène publiée dans la revue Nova Francia, vol. 4 et 5, (1929-1935).

21. Jugements et délibérations du Conseil Souverain, vol. 4, p. 304-505.

22. Juchereau et Duplessis, Annales, p. 46. 
En effet, vers la fin du XVIII ${ }^{\mathrm{e}}$ siècle surtout, seuls les proches des insensés (ou les insensés eux-mêmes, lorsqu'on les interroge) qui, en raison de leur proximité avec les victimes sont en mesure d'appuyer leur diagnostic sur des observations concrètes, imputent encore le désordre mental à de mauvaises expériences ou à des traumatismes émotionnels vécus par la personne en question.

L'histoire de Marianne D., à titre d'exemple, est intéressante à cet égard. En 1787. elle déclare qu'il y a environ 5 ans «quelques-uns de ses parents ayant pris pour démence son affliction où elle était tombée suite à la mort de son mari», elle s'était vue frappée d'interdiction, alors que de son côté, le «savant» curé de sa paroisse optait plutôt pour l'explication «scientifique» et déclarait que, selon lui, cette dernière avait plutôt «été battue de vapeurs qui la rendaient presqu'à demi-folle ${ }^{23}$ ».

Donc, probablement parce que l'explication «psychologique» n'apparaît pas assez concrète aux yeux de gens instruits, plusieurs ont de plus en plus tendance à reléguer aux oubliettes l'observation du passé émotif des victimes pour ne se préoccuper que de déductions basées sur leur histoire médicale ce qui, bien sûr, liait toujours plus la folie aux médecins et à leurs médecines.

Car, à ce moment, les médecins sont presque unanimes à déclarer que la folie puise ses origines dans des problemes de nature organique, cherchant chez leurs patients des déterminants ou des symptômes de la folie de nature physiologique. On privilégie à ce moment l'affaiblissement du cerveau et la tension des nerfs, qu'elle soit trop forte ou trop lâche. Cette tendance à médicaliser, et donc à médicamenter la folie, bien loin de s'estomper avec le XIX ${ }^{e}$ siècle, allait plutôt se confirmer et se généraliser.

Pourtant, la première moitié du XIX ${ }^{\mathrm{e}}$ siècle est bien celle où Philippe Pinel publie son célèbre Traité médico-philosophique qui jetait les bases théoriques d'une thérapie travaillant au niveau des émotions, à l'origine des désordres mentaux selon l'aliéniste, retournant ainsi aux sources de la connaissance populaire. C'est en effet au début de ce siècle que remonte l'émergence du fameux traitement moral (dit moral par opposition à physique ou, si l'on préfère, traitement du moral), destiné à traiter l'équilibre émotionnel du patient plutôt que son corps.

Parce qu'elle se réclamera du traitement moral lors de la promotion de l'asile au milieu du siècle, on aurait pu s'attendre à ce que la médecine d'ici emboîte le pas à la révolution pinélienne. Mais, si l'on prend la peine

23. ANQ-Q, Levée d'interdiction de Marianne D., 2 février 1787. 
d'analyser la pratique quotidienne du corps médical québécois, force est d'admettre que tel ne fut pas le cas.

Nous avons déjà présenté ailleurs la documentation d'interdiction et de curatelle et l'on sait la richesse des renseignements qu'elle renferme sur tous les aspects de la vie du fou qui se trouvait privé, par ce processus légal, de la gérance de ses biens ${ }^{24}$. Or, cette documentation d'interdiction et de curatelle est précieuse pour la connaissance des causes présumées de la folie, puisqu'on y trouve des témoignages et les commentaires des médecins et des proches des victimes en ce qui a trait au trouble mental dont souffraient ces dernières.

Les 489 dossiers d'interdiction consultés pour les 45 premières années du XIX ${ }^{e}$ siècle $^{25}$ ont livré 92 causes répertoriées selon le type d'individu donnant ainsi son avis, qu'il soit membre de la profession médicale ou simple citoyen, de même que selon le type de cause présumée (physique ou «psychologique»). Bien que le total de ces causes demeure assez limité, il est toutefois suffisant pour permettre de dégager certaines tendances qui ne trompent pas.

Tableau 1

Causes présumées de la folie au sein de l'interdiction et de la curatelle, 1801-1845

\begin{tabular}{|l|c|c|c|}
\hline \multicolumn{1}{|c|}{ Causes présumées } & $\begin{array}{c}\text { Diagnostics de } \\
\text { médecins }\end{array}$ & $\begin{array}{c}\text { Diagnostics de } \\
\text { non- } \\
\text { professionnels }\end{array}$ & Total \\
\hline $\begin{array}{l}\text { Causes de nature } \\
\text { physique }\end{array}$ & 36 & 41 & 77 \\
\hline $\begin{array}{l}\text { Causes de nature } \\
\text { "psychologique" }\end{array}$ & 1 & 14 & 15 \\
\hline Total & 37 & 55 & 92 \\
\hline
\end{tabular}

Sources: Archives de la curatelle, 1801-1845, ANQ-Q : ANQ-M.

Constatation importante, alors que les causes présumées de nature «psychologique» représentent de loin la majorité des causes exprimées au $\mathrm{XVII}^{\mathrm{e}}$ siècle, on ne retrouve plus, sur ces 92 causes, que 15 cas où l'on impute le désordre mental au traumatisme émotionnel. Tout aussi significatif: une seule des causes de nature psychologique est émise par un médecin, ce qui signifie qu'à 36 reprises sur 37 les membres de cette profession

24. A.Cellard, Histoire Sociale/Social History, vol. XIX, $\mathrm{n}^{\circ} 38$ (nov. 1986) p. 443 450.

25. A. Cellard, Histoire de la folie au Québec de 1600 à I850, Montréal, Boréal, chap. 3. 
attribuent une origine strictement physiologique ou organique au désordre de leur patient.

Ainsi, 14 des 55 causes exprimées par la population - parents, amis, voisins - sont encore attribuées au désordre «psychologique». On dira, par exemple, de Joseph L. «que son absence d'esprit est causée je crois par le chagrin qu'il a eu de la perte de sa mère ${ }^{26}$ ». L'épouse d'Augustin T., elle. nous apprend que ce dernier est trouble parce qu'on lui reproche d'avoir payé sa maison trop cher. Lorsque le juge qui procède à l'interdiction lui laisse la parole. Augustin T., comme d'autres, abonde dans le même sens: «J'ai essuyê de grandes pertes dernièrement qui m'ont dérangé. Je ne puis plus conduire mes affaires, tout me paraît en désordre ${ }^{27}$ ».

Mais, probablement parce qu'il ne paraît plus savant ni instruit d'attribuer la folie au désordre émotionnel, 77 des causes exprimées sont imputées à un problème d'origine physique, de l'épilepsie à la maladie cardiaque. Même dans les cas où l'on semble convaincu de l'origine psychologique du mal, certains donnent l'impression de se sentir obligés d'ajouter le terme «maladie» aux causes présupposées de la folie. Ainsi, à titre d'exemple, l'épouse de Jean-Baptiste M. dit de lui qu'il « est malade, bien qu'elle croie que sa maladie est occasionnée par la jalousie, que depuis qu'il est malade, il a l'esprit aliéné ${ }^{28}$ ». Un proche de Louis C., quant à lui, affirme «qu'il ne connaît personnellement aucune cause qui ait pu produire la maladie du dit Louis $C$. si ce n'est que la peur du cholera et la mort de Dame veuve Thibodeau qui l'avaient affecté ${ }^{29} »$. De fait, certaines personnes semblent se contraindre à faire mention d'une cause médicale, aussi vague soit-elle, de crainte de paraître ignares. Par exemple, de Marie C., un parent dit qu'elle est dans «un état de faiblesse d'esprit dû à la mort de son mari et une maladie ${ }^{30} »$, se croit-il obligé d'ajouter.

Il n'y a pas de confusion chez les médecins, cependant, puisqu'à une exception près ils sont unanimes à imputer au malfonctionnement du corps la source du désordre mental, comme on l'a vu tantôt. Ils accusent les nerfs, la constipation, la paralysie, l'alcool et autres facteurs ${ }^{31}$. Ils sont à ce point convaincus de l'origine organique du mal qu'ils préfèrent parfois ignorer les observations des proches des victimes pour mettre de l'avant des explications de nature physiologique. Par exemple, le père de Gabriel $\mathrm{C}$. dit de

26. ANQ-Q, Interdiction et curatelle de Joseph L., 15 avril 1820.

27. ANQ-Q, Interdiction et curatelle d'Augustin T., $1^{\text {er }}$ mars 1816.

28. ANQ-M, Interdiction et curatelle de Jean-Baptiste M., 6 mai 1817.

29. ANQ-Q, Interdiction et curatelle de Louis C., 29 août 1834.

30. ANQ-M. Interdiction et curatelle de Marie C., 22 mai 1827.

31. Cellard, A., Histoire de la folie..., op. cit., p. 155-56. 
son fils «que l'absence de son esprit provient en grande partie de petits chagrins de ménage» et qu'en ce moment «le dit Gabriel C. fils se livre à des excès de débauche et à l'ivrognerie». Ne retenant que le symptôme, semble-t-il, le $\mathrm{D}^{r}$ Fisher affirme plutôt que «son dérangement, à mon opinion, provient de l'ivrognerie $\mathrm{e}^{32} »$.

Bien qu'elle tende à disparaître rapidement, c'est donc, paradoxalement, dans la population que survit encore l'explication «psychologique» de la folie en cette première moitié du XIX ${ }^{\mathrm{e}}$ siècle. C'est que, malgré les «découvertes» de Pinel, la médecine ne se laissera pas facilement convaincre que l'origine du désordre mental n'est pas nécessairement organique, ce qui viendrait du coup invalider sérieusement sa prétendue expertise dans un champ de la santé aussi important. L'avènement du positivisme aidant, les médecins n'étaient peut-être pas prêts à assimiler ce qu'il y avait de vraiment important dans la thèse de Pinel. Travaillant à partir d'éléments concrets du mal, les symptômes, ils allaient utiliser une médication concrète: saignées, purgations, irritants, toniques, fortifiants, tranquillisants, diètes, douches, bains froids, bref, autant d'artifices destinés à agir autant sur les symptômes đu mal que sur le mal lui-même.

Dans un autre ordre d'idé, il convient de souligner que ce ne sont pas n'importe quels médecins qui intervenaient alors auprès des insensés, mais bien les plus fameux d'entre eux, lesquels, d'ailleurs, jouèrent un rôle des plus importants dans la mise en place, dans la première moitié du siècle, de structures publiques destinées au soin des aliénés et dont ils étaient d'ailleurs les principaux bénéficiaires.

En effet, si à 37 reprises les médecins donnent leur interprétation des causes de la folie au sein de l'interdiction et de la curatelle, c'est près d'une centaine d'interventions au total auprès d'interdits qu'il nous a été en mesure de retracer au sein de cette documentation, ce qui permet d'esquisser un profil de l'expertise québécoise en matière d'aliénation mentale dans la première moitiê du XIX ${ }^{\mathrm{e}}$ siècle.

En tête de liste, on retrouve sans surprise Joseph Morrin, avec une dizaine de récurrences. Il est à souligner que ce dernier fut, dans les années 1830, commissaire aux aliénés et co-fondateur (et co-propriétaire) de l'asile de Beauport. Suivent, dans l'ordre, les docteurs Painchaud, collaborateur de Morrin et de Douglas, à qui on proposa le poste de surintendant de Beauport, où il siégea d'ailleurs à titre de commissaire des aliénés. Puis, James Douglas, bien sûr, principal promoteur de Beauport; James Fisher, médecin responsable des aliénés à l'Hôpital général de Québec, puis

32. ANQ-Q, Interdiction et curatelle de Gabriel C., 1810. 
Marsden, Arnoldi, Longmore, Shelby, Robertson, Crawford, Thresler, Stephenson qui, tous, furent soit commissaires aux aliénés, promoteurs d'institutions pour lunatiques et qui, surtout, comptent parmi les membres les plus émérites de la profession. Ces praticiens, pour la plupart natifs de Grande-Bretagne allaient donc tenter de développer ici le lucratif lunacy trade anglais, ce qui se concrétisera dans le second quart du XIX ${ }^{\mathrm{e}}$ siècle avec l'ouverture des premiers asiles sous contrôle médical. C'est finalement à cette époque, dans les perceptions comme dans la pratique, que le fou allait devenir un «malade» à part entière.

\section{CONCLUSION}

Portée par des progrès bien réels, la médecine officielle allait rapidement étendre au XIX ${ }^{\mathfrak{e}}$ siècle sa mainmise sur la plupart des domaines liés aux soins de la santé. C'est cependant sans avoir à faire preuve de quelque compétence que ce soit au niveau thérapeutique qu'elle allait, entre les $\mathrm{XVII}^{\mathfrak{e}}$ et $\mathrm{XIX}^{\mathrm{e}}$ siècles, s'accaparer de l'important champ de la folie en imposant son point de vue quant aux causes des désordres mentaux. Cela bien sûr ne se fit pas du jour au lendemain. C'est petit à petit que l'on observe cette évolution sur deux siècles et demi d'histoire, à mesure que progressaient, parmi les couches populaires dites inférieures, la culture de l'élite et, parmi la population, la confiance dans les moyens de la science en général et de la médecine en particulier.

Marginale au XVII ${ }^{\mathrm{e}}$ siècle, l'idée que la folie origine avant tout d'un désordre physique ou biologique allait graduellement déloger la croyance populaire qui liait l'aliénation mentale à des traumatismes émotionnels et rallier la majorité au début du XIX ${ }^{\mathrm{e}}$ siècle.

C'est donc complètement à l'inverse du postulat traditionnel d'une médecine scientifique progressiste éclairant les noirceurs de la masse qu'allait fonctionner la dynamique selon laquelle, pour le meilleur et pour le pire, furent remis dans les mains des médecins et de leurs traitements, le sort de générations entières d'insensés. 